\title{
The X-ray afterglow of the short gamma ray burst 050724
}

\author{
S. Campana ${ }^{1}$, G. Tagliaferri ${ }^{1}$, D. Lazzati ${ }^{2}$, G. Chincarini ${ }^{1,3}$, S. Covino ${ }^{1}$, K. Page $^{4}$, P. Romano $^{1}$, A. Moretti $^{1}$, \\ G. Cusumano ${ }^{5}$, V. Mangano ${ }^{5}$, T. Mineo ${ }^{5}$, V. La Parola ${ }^{5}$, P. Giommi ${ }^{6}$, M. Perri ${ }^{6}$, M. Capalbi ${ }^{6}$, B. Zhang ${ }^{7}$, \\ S. Barthelmy ${ }^{8}$, J. Cummings ${ }^{8}$, T. Sakamoto ${ }^{8}$, D. N. Burrows ${ }^{9}$, J. A. Kennea ${ }^{9}$, J. A. Nousek ${ }^{9}$, \\ J. P. Osborne ${ }^{4}$, P. T. O’Brien ${ }^{4}$, O. Godet ${ }^{4}$, and N. Gehrels ${ }^{8}$
}

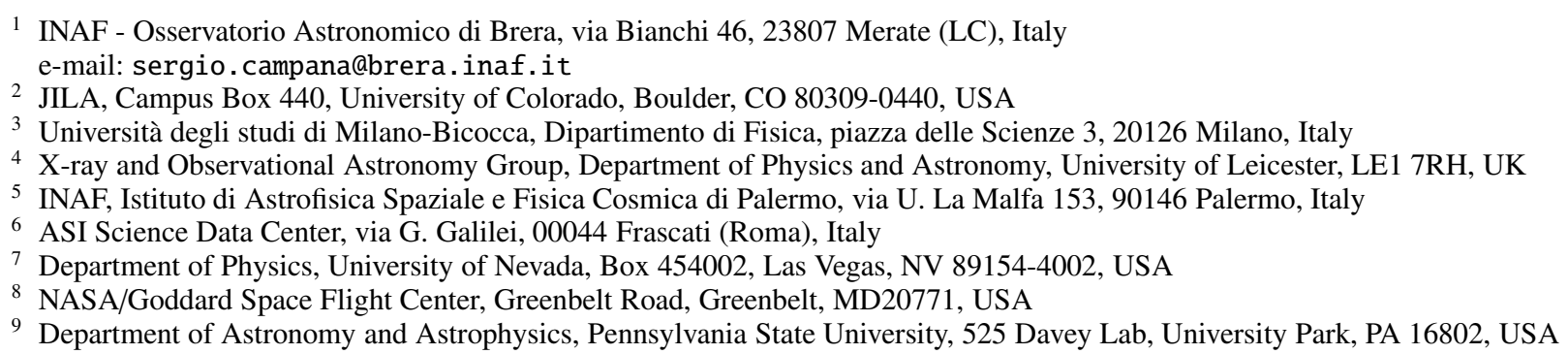

Received 13 January 2006 / Accepted 14 March 2006

\section{ABSTRACT}

\begin{abstract}
Short duration ( $\$ 2 \mathrm{~s})$ Gamma-ray bursts (GRBs) have been a mystery since their discovery. Until May 2005 very little was known about short GRBs, but this situation has changed rapidly in the last few months since the Swift and HETE-2 satellites have made it possible to discover X-ray and optical counterparts to these sources. Positional associations indicate that short GRBs arise in close-by galaxies $(z<0.7)$. Here we report on a detailed study of the short GRB 050724 X-ray afterglow. This burst shows strong flaring variability in the X-ray band. It clearly confirms early suggestions of X-ray activity in the 50-100 s time interval following the GRB onset seen with BATSE. Late flare activity is also observed. These observations support the idea that flares are related to the inner engine for short GRBs, as well as long GRBs.
\end{abstract}

Key words. gamma rays: bursts - X-rays: general

\section{Introduction}

Gamma Ray Bursts (GRBs) are intense flashes of $\gamma$-ray radiation outshining all other sources in the gamma-ray sky. GRBs can be divided into (at least) two classes based on their temporal and spectral properties: short and long GRBs. Short GRBs have a typical duration of $0.2 \mathrm{~s}$ and can last from a few milliseconds to several seconds. Short GRBs are harder (i.e. have more flux at higher energies) than long bursts. Thus, in a plane $\left[T_{90}\right.$ - hardness ratio] they lie in a region mostly distinct from long GRBs (Kouveliotou et al. 1993). A more general definition of short burst has been given by Norris \& Bonnell (2006) that the initial spike exhibits negligible spectral evolution at energies above $\sim 25 \mathrm{keV}$. Short GRBs comprise about $25-30 \%$ of the BATSE $(25-350 \mathrm{keV})$ sample and about $12 \pm 4 \%$ of the Swift sample. This lower fraction is due to the softer energy band (15-150 keV) of the Burst Alert Telescope (BAT, Barthelmy et al. 2005a) onboard Swift (Gehrels et al. 2004).

Despite the great improvements in our knowledge of long GRBs in the last decade, very little was known on short GRBs. This situation changed drastically starting from May 2005, when the Swift satellite (Gehrels et al. 2004) was able to detect and accurately localize for the first time the X-ray afterglow of the short burst GRB 050509B (Gehrels et al. 2005). This burst lies near a luminous, non star-forming $\left(<0.1 M_{\odot} \mathrm{yr}^{-1}\right)$ elliptical galaxy at $z=0.22$ located in a cluster of galaxies. The a posteriori probability of the position being close to such a nearby, luminous galaxy by coincidence is $\sim 10^{-4}$ (fainter objects in the error circle have been reported by Bloom et al. 2006). Two months later the HETE-2 satellite localized GRB 050709, the first short GRB with an optical counterpart. The object was coincident with a weak X-ray source and was located inside a galaxy at $z=0.16$ (Covino et al. 2006; Hjorth et al. 2005; Fox et al. 2005). Spectroscopic observations showed that the dominant stellar population is relatively young $(\sim 1 \mathrm{Gyr})$ and that there is ongoing star formation $\left(\sim 0.2 M_{\odot} \mathrm{yr}^{-1}\right.$, Fox et al. 2005; Covino et al. 2006). GRB 050724 was the first short GRB with low energy prompt X-ray emission lasting for $100 \mathrm{~s}$ after the main short pulse, strong early X-ray afterglow and an unusual X-ray re-brightening at $3 \times 10^{4} \mathrm{~s}$ (a short account of these data has been given by Barthelmy et al. 2005b). The X-ray and optical afterglow of this burst (D'Avanzo et al. 2005; Gal-Yam et al. 2005; Barthelmy et al. 2005b; see also Covino et al. 2005) is located off-center in an elliptical galaxy at $z=0.258$ with a very low star-formation ( $<0.02 M_{\odot} \mathrm{yr}^{-1}$; Prochaska et al. 2005). The X-ray afterglow of the short GRB 050813 simply faded without any flare, leading to a localization consistent with a distant $(z \sim 0.7)$ cluster of galaxies (Gladders et al. 2005). The host galaxy for this burst has not been found yet (Prochaska et al. 2006; see Moretti et al. 2006, for an updated position). 
Table 1. Observation log.

\begin{tabular}{ccccc}
\hline \hline OBS.ID. & $\begin{array}{c}\text { Start time } \\
(\mathrm{s})^{*}\end{array}$ & Mode & $\begin{array}{c}\text { Exp. time } \\
(\mathrm{s})\end{array}$ & $\begin{array}{c}\text { Count rate } \\
3 \sigma \mathrm{UL}\end{array}$ \\
\hline 00147478000 & 74 & WT & 8438 & \\
& 189 & PC & 27737 & \\
00147478001 & 127716 & PC & 16947 & \\
00147478002 & 215626 & PC & 135 & $<1.1 \times 10^{-1}$ \\
00147478003 & 215679 & PC & 2121 & $<6.2 \times 10^{-3}$ \\
00147478004 & 377054 & PC & 5244 & $<2.8 \times 10^{-1}$ \\
00147478005 & 377083 & PC & 2218 & $<5.8 \times 10^{-3}$ \\
00147478006 & 388061 & PC & 267 & $<1.6$ \\
$00147478007^{+}$ & 388119 & PC & 25979 & $<1.0 \times 10^{-3}$ \\
00147478008 & 474403 & PC & 1383 & $<1.1 \times 10^{-2}$ \\
$00147478009^{+}$ & 474582 & PC & 21129 & $<9.2 \times 10^{-4}$ \\
\hline
\end{tabular}

* Time from the BAT trigger time.

+ These two observations when summed together lead to a (low significance) detection at a rate of $(2.6 \pm 1.1) \times 10^{-4} \mathrm{c} \mathrm{s}^{-1}$.

In addition, GRB 050724 is the first and only GRB for which a radio afterglow has been detected (Soderberg et al. 2005).

In this paper we present a thorough analysis of the X-ray properties of GRB 050724 as observed by Swift. In Sect. 2 we present the data and in Sect. 3 their spectral and temporal analysis. Section 4 is dedicated to the discussion and Sect. 5 to the conclusions.

\section{Swift data}

GRB 050724 (Covino et al. 2005) was discovered at 2005-07-24 12:34:09 UT by the BAT. Swift slewed to the burst position in only 74 s. The X-ray Telescope (XRT, Burrows et al. 2005a) observations started at 12:35:22.9 UT in Auto State. XRT detected a rapidly fading source (Romano et al. 2005a). The UltraViolet and Optical Telescope (UVOT, Roming et al. 2005) did not detect the afterglow $(V>18.8$, Chester et al. 2005).

The XRT observed the GRB position nine times. The log of these observations is presented in Table 1. The first observation is split into two parts with two different observing modes. For bursts observed in Auto State the XRT observations start in Window Timing (WT) mode (providing just 1D imaging) and when the source gets below a predefined count rate threshold XRT switches automatically to the more common Photon Counting mode (PC, providing 2D imaging and full spectroscopic resolution; for a description of XRT observing modes, see Hill et al. 2004). Cross-calibration between modes assures that the two modes, PC and WT, provide the same rate (within a few percent) on steady sources.

GRB 050724 was detected in the first two observations and in the combination of observation 7 and 9 (47 108 s). In the following, we do not consider the other observations. Finally, we note that a dust-scattered X-ray halo has been discovered around GRB 050724 (Romano et al. 2005b). This topic has been fully covered by Vaughan et al. (2006) and it will not be discussed in the present work.

\section{Data analysis}

\subsection{Burst Alert Telescope}

The BAT light curve shows a short bright peak with $0.25 \mathrm{~s} \mathrm{du}-$ ration. There is additional prompt emission in the time interval $[-20,20] \mathrm{s}$ with a $T_{90}$ of $3.0 \pm 1.0 \mathrm{~s}$ (Barthelmy et al. 2005b).
Besides the short spike, there is an extended low-flux tail lasting for $\gtrsim 200 \mathrm{~s}$ (Krimm et al. 2005). If this extended tail is included, the duration becomes $T_{90}=152.4 \pm 9.2 \mathrm{~s}$. Since this $T_{90}$ is longer than the canonical $2 \mathrm{~s}$, a more general definition of short burst has been suggested by Norris \& Bonnell (2006) based on the unique properties of the initial spike: this spike should exhibit negligible spectral evolution at energies above $\sim 25 \mathrm{keV}$. The first peak is relatively hard with a power law photon index $\Gamma=1.71 \pm 0.16$ (90\% confidence level, throughout the paper), after which the emission softens to $\Gamma=2.5 \pm 0.2$. The peak $15-350 \mathrm{keV}$ fluence $(1 \mathrm{~s})$ is $(2.6 \pm 0.3) \times 10^{-7} \mathrm{erg} \mathrm{cm}^{-2}$ (in the $T_{90}$ is $\left.(3.9 \pm 1.0) \times 10^{-7} \mathrm{erg} \mathrm{cm}^{-2}\right)$ whereas the total fluence is $(1.5 \pm 0.3) \times 10^{-6} \mathrm{erg} \mathrm{cm}^{-2}$ (see also Barthelmy et al. 2005b). From the BAT analysis it is clear that the bulk of the energy is not emitted in the short initial spike $\left(<1 \mathrm{~s}\right.$ or $\left.T_{90}\right)$ but in the extended tail. Moreover, the bump at $50-100 \mathrm{~s}$ is consistent in time and spectrum with the soft excess observed with BATSE after summing a large number of bright short bursts (Lazzati et al. 2001).

\subsection{X-ray telescope}

All data were processed with the standard XRT pipeline within FTOOLS 6.0 (xrtpipeline v. 0.8.8) in order to produce screened event files. WT data were extracted in the $0.5-10 \mathrm{keV}$ energy range, $\mathrm{PC}$ data in the $0.3-10 \mathrm{keV}$ range. Standard grade filtering was adopted ( $0-2$ for WT and $0-12$ for PC, according to XRT nomenclature, see Burrows et al. 2005a). From these data we extracted spectra and light curves using regions selected to avoid pile up and to maximize the signal to noise (see Table 1 and below). In WT mode we adopted the smaller than standard extraction region of $20 \times 20$ pixels $\left(47^{\prime \prime} \times 47^{\prime \prime}\right)$ along the WT line in order to avoid any small contribution from the dust-scattered halo (Vaughan et al. 2006). In PC we used a small circular extraction region of 10 arcsec. Appropriate ancillary response files were generated with the task xrtmkarf, accounting for Point Spread Function (PSF) corrections. The latest response matrices (v.007) were used.

\subsection{Image analysis}

An initial source position has been reported in Barthelmy et al. (2005b). We take advantage of the second observation where the pile-up is completely absent and there is no contamination from the dust-scattered halo. Using the FTOOLS task xrtcentroid, which includes also systematic effects, we derive $\mathrm{RA}(\mathrm{J} 2000)=16^{\mathrm{h}} 24^{\mathrm{h}} 44.40^{\mathrm{s}}, \operatorname{Dec}(\mathrm{J} 2000)=-27^{\circ} 32^{\prime} 27.4^{\prime \prime}$ with a $90 \%$ error radius of $4.2^{\prime \prime}$. This includes a correction for the satellite boresight (Moretti et al. 2006). This improved XRT position is $1^{\prime \prime}$ from the previous determination (Barthelmy et al. 2005b) and 0.5" and 0.4" from the Chandra and optical (VLT, D'Avanzo et al. 2005; Barthelmy et al. 2005b), and VLA positions (Soderberg 2005), respectively.

\subsection{Timing analysis}

The $0.2-10 \mathrm{keV}$ light curve of the GRB 050724 afterglow is shown in Fig. 1. The light curve is in the GRB rest-frame and the effective energy range is $0.25-12.6 \mathrm{keV}$. The early part of the curve comes from the BAT data extrapolated to the XRT energy band with the BAT spectral model described above. The second part is from the XRT data. Fluxes have been computed according to the best fit models (see below). It is clear from Fig. 1 that the 


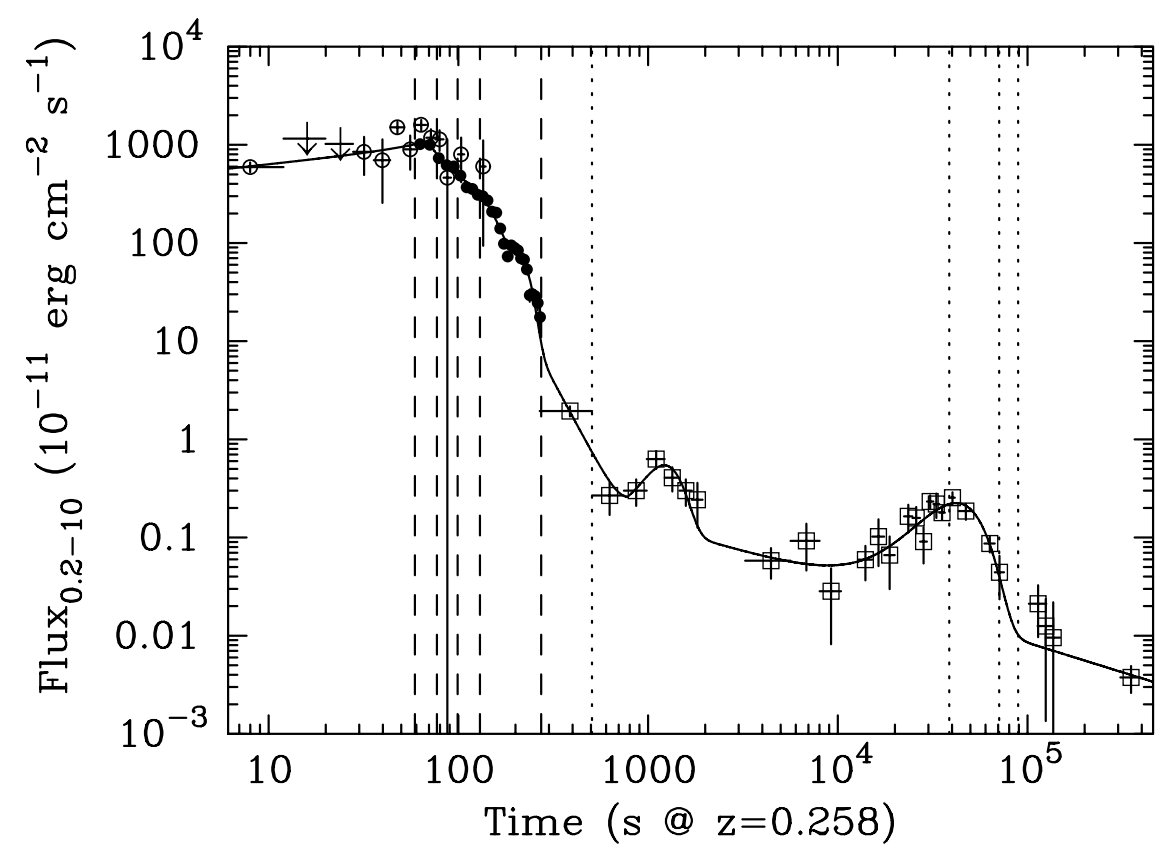

Fig. 1. X-ray light curve of GRB 050724. Count rates have been converted into $0.2-10 \mathrm{keV}$ unabsorbed flux after spectral modelling (see text). Times have been converted to the GRB progenitor reference time. Open circles mark BAT data (as well as $3 \sigma$ upper limits) extrapolated to the $0.2-10 \mathrm{keV}$ energy band; filled circles mark XRT-WT data and open squares XRT-PC data, respectively. The continuous line represents the best fit model obtained with four Gaussians and a triple power law (see text). Dashed lines indicate WT mode time intervals selected for spectral analysis. Dotted lines indicate time intervals used for spectral analysis in PC mode.

light curve is extremely rich. There is a steep decay following the GRB proper (possibly with some structure overlayed) and two big flares. We fit this light curve with a doubly-broken power law (as usually done for long GRBs as well, see Chincarini et al. 2005; Nousek et al. 2006; O’Brien et al. 2006) plus four Gaussians. Formally this model does not provide a good representation of the data since we have a reduced $\chi_{\text {red }}^{2}=2.5$ (with 47 degrees of freedom, d.o.f.), but it catches the main features of the afterglow light curve (see Fig. 1, the high $\chi^{2}$ is mainly due small scale fluctuations). The first power law has a rising index $\alpha_{1}=+0.2 \pm 0.1$ and accounts for the prompt emission observed by the BAT (lack of data in this first part is due to upper limits in the BAT light curve). The first time break is at $t_{1}=69 \pm 2 \mathrm{~s}$, indicating when the very steep decay starts. The second power law has $\alpha_{2}=-3.6 \pm 0.1$. Then the power law decay flattens to $\alpha_{3}=-0.6 \pm 0.2$ after $t_{2}=775 \pm 395 \mathrm{~s}$. During the first $\sim 1000 \mathrm{~s}$ the source fades by about four orders of magnitude in flux. On top of this behaviour we have to include at least four main (Gaussian) flares with start time $109 \pm 7,215 \pm 5,1226 \pm 165$ and $42100 \pm 2230 \mathrm{~s}$, respectively ${ }^{1}$. The widths (Gaussian $\sigma$ ) of the four flares are $40 \pm 8 \mathrm{~s}, 25 \pm 6 \mathrm{~s}, 263 \pm 194$ and $14590 \pm 2425 \mathrm{~s}$, respectively $(\delta t / t$ of the flares are $0.37 \pm 0.08,0.12 \pm 0.03$, $0.21 \pm 0.16$ and $0.35 \pm 0.02$ ). Major Gaussian-like flares have been reported in GRB 050502B (Burrows et al. 2005b; Falcone et al. 2006) and XRF 050406 (Romano et al. 2006). We also investigate different models for the first two flares. An exponential tail or a Lorentzian model provide worse fits than a Gaussian model. After MacFadyen et al. (2005) we also consider a fast rise exponential decay (FRED) for the first two flares ${ }^{2}$. The fit is slightly improved $\left(\chi_{\text {red }}^{2}=2.2\right.$, with 45 d.o.f., $2.5 \sigma$ according to

\footnotetext{
${ }^{1}$ The statistical significance of the third flare is about $2.2 \sigma$ computed in the 300-2000 s time interval.

2 The FRED flares have been modelled as $x^{r} \times \exp -\left(t-t_{0}\right) / t_{\mathrm{c}}$, where $t$ is the time, $t_{0}$ is the start time, $t_{\mathrm{c}}$ is decay time and $x=2.71\left(t-t_{0}\right) /\left(r t_{\mathrm{c}}\right)$, accounts for the rise. The peak in this parametrization is at $r t_{\mathrm{c}}-t_{0}$.
}

an F-test). The peaks of the two flares in this case move to $112 \mathrm{~s}$ and $187 \mathrm{~s}$.

From the theoretical point of view, flares are sometimes modelled with power laws (e.g. Zhang et al. 2006). We tried smoothly joined power laws to describe the flares on top of the doubly-broken power law. The fit provides a slightly better description of the data with $\chi_{\text {red }}^{2}=2.3$ (46 d.o.f.). The times of the two breaks are at $t_{1}=70 \pm 2 \mathrm{~s}$ and $t_{2}=820 \pm 180 \mathrm{~s}$ and the power law indices are $\alpha_{1}=0.2 \pm 0.1, \alpha_{2}=-3.6 \pm 0.2$ and $\alpha_{3}=-0.6 \pm 0.2$. Peak time of the four flares are $148 \pm 7 \mathrm{~s}$, $210 \pm 9 \mathrm{~s}, 994 \pm 106$ and $44478 \pm 9298 \mathrm{~s}$ (here and in the following times are rest-frame). The rising exponents (with reference to the BAT trigger) are not well constrained, the one relative to the first flare is very flat (fixed to 1 ), the following two are very steep (fixed to 10) and the last one is $2.2 \pm 0.7$. The decaying exponents are better constrained and all very steep: $9 \pm 3,11 \pm 4,3_{-1}^{+3}$ and $4_{-2}^{+3}$, respectively. A model with four flares without the underlying broken power law provides a worse fit with $\chi_{\text {red }}^{2}=3.6$.

The total fluence of the GRB 050724 afterglow in the $0.2-10 \mathrm{keV}$ energy band is $1.4 \times 10^{-6} \mathrm{erg} \mathrm{cm}^{-2}$ in the $10-10^{6} \mathrm{~s}$ time interval. This is larger than the burst prompt fluence $(1 \mathrm{~s}$, extrapolated to the same band) by a factor of $\sim 10$. The fluence of the first flare is $2.5 \times 10^{-7} \mathrm{erg} \mathrm{cm}^{-2}$ ( $21 \%$ of the total fluence). The fluence of the second flare is $2.8 \times 10^{-8} \mathrm{erg} \mathrm{cm}^{-2}$ ( $2 \%$ of the total). Results are similar (within a few percent of the total fluence) also in the case of a FRED modelling. The last two flares have $2.8 \times 10^{-9} \mathrm{erg} \mathrm{cm}^{-2}(0.2 \%)$ and $7.7 \times 10^{-8} \mathrm{erg} \mathrm{cm}^{-2}(7 \%)$, respectively. Flares account for $\sim 30 \%$ of the total fluence in the $0.2-10 \mathrm{keV}$ energy band.

\subsection{Spectral analysis}

Given the complex behaviour of the light curve we performed the spectral analysis over different segments of the light curve. A first indication of spectral changes can be obtained from a band 


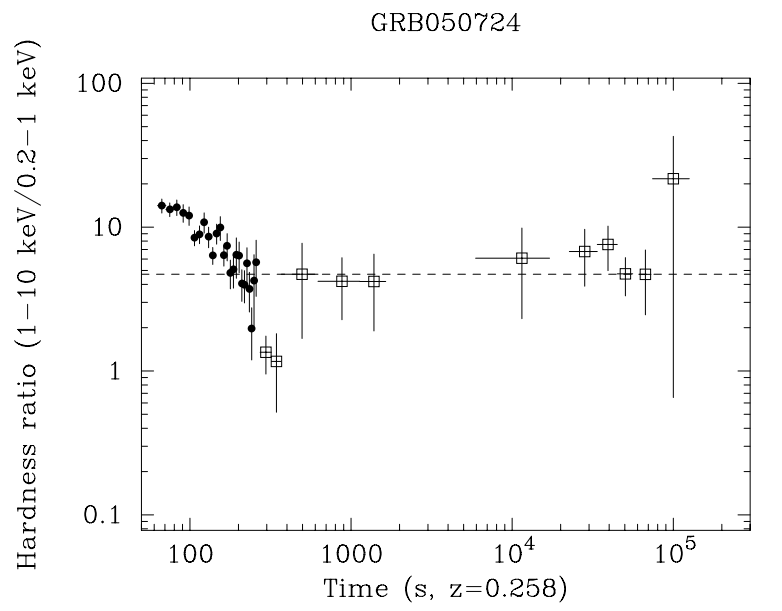

Fig. 2. Hardness ratio light curve. The ratio is between $1-10 \mathrm{keV}$ to $0.2-1 \mathrm{keV}$. Time has been corrected for redshift time dilation. Filled circles mark XRT WT data, open squares XRT PC data, respectively. The dashed line indicates the mean value of the data starting from $370 \mathrm{~s}$ from the burst trigger (rest frame).

(hardness) ratio analysis (we used the $1-10 \mathrm{keV}$ over $0.2-1 \mathrm{keV}$ energy bands, Fig. 2). It is apparent that the rapid decay up to $500 \mathrm{~s}$ after the burst shows a transition from hard to soft. After $\sim 500 \mathrm{~s}$ the hardness ratio becomes stable, despite the presence of the two late flares (rest frame time $t>500 \mathrm{~s}$ ). The rise in hardness ratio after the flare is uncommon: in other GRBs the ratio typically returns to a low level after each flare.

For a proper spectral fitting, we divided the first part of the XRT light curve into five intervals containing at least 500 counts. The first four are in WT mode and the last one is in PC mode. The data were rebinned to have 30 counts per energy bin. The $\mathrm{X}$-ray absorption has been modelled with a constrained Galactic component (3.4-4.2 $\times 10^{21} \mathrm{~cm}^{-2}$, Vaughan et al. 2006), plus an intrinsic $(z=0.258)$ column density. The model used within XSPEC (11.3.2) is tbabs. For the spectrum we adopted a simple power law.

The overall fitting is good, providing a reduced $\chi^{2}=1.03$ (321 degrees of freedom and 36\% null hypothesis probability). The intrinsic column density is constant within the errors. As can be seen from the hardness ratio plot and from the detailed spectral analysis (Table 2) there is a clear trend from hard to soft in the first $400 \mathrm{~s}$ (rest frame) after the burst. This is observable in the power law photon index which is softening from $1.4 \pm 0.1$ to $3.3 \pm 0.4$.

We also analyzed the last part of the light curve adopting a different approach. In this case the number of photons is relatively low and we fit an absorbed (Galactic plus local) power law using Cash-statistics (see second part of Table 2). The first PC interval is at the end of the second part (the steepest) of the 3 -power law component and well after the first two flares. The spectrum during this interval is very soft $(\Gamma=3.3 \pm 0.4$. The remaining three intervals instead have very similar spectra, somewhat harder than the first PC interval.

Given the complex behaviour of the X-ray afterglow we also tried a cut-off power law model since this model can accommodate spectral variations as demonstrated in the case of GRB 050502B's flare (Falcone et al. 2006). The fit confirms the results above with cut-off energies decreasing with time (the overall $\chi_{\text {red }}^{2}=1.01$ but the chance improvement computed with an F-test is just 5\%).
Table 2. Spectral fits.

\begin{tabular}{cccc}
\hline \hline $\begin{array}{c}\text { Interval } \\
\text { (s, rest frame) }\end{array}$ & $\begin{array}{c}N_{\mathrm{H}} @ z \\
\left(10^{21} \mathrm{~cm}^{-2}\right)\end{array}$ & $\begin{array}{c}\text { Power law } \\
\Gamma\end{array}$ & $\begin{array}{c}\text { Mean flux } \\
(0.5-10 \mathrm{keV})\end{array}$ \\
\hline $59-77$ & $2.3_{-1.2}^{+2.0}$ & $1.41_{-0.09}^{+0.09}$ & $9.3 \times 10^{-9}$ \\
$77-99$ & $4.0_{-1.3}^{+2.0}$ & $1.84_{-0.10}^{+0.11}$ & $6.0 \times 10^{-9}$ \\
$99-130$ & $2.3_{-1.1}^{+1.8}$ & $1.91_{-0.10}^{+0.11}$ & $4.7 \times 10^{-9}$ \\
$130-268$ & $1.9_{-0.8}^{+1.5}$ & $2.34_{-0.09}^{+0.10}$ & $8.1 \times 10^{-10}$ \\
$268-89232$ & $<0.9^{\#}$ & $1.98_{-0.20}^{+0.16}$ & $1.4 \times 10^{-12}$ \\
\hline $268-506^{*}$ & $<0.7^{+}$ & $3.30_{-0.36}^{+0.41}$ & $2.3 \times 10^{-11}$ \\
$507-38647$ & & $1.76_{-0.17}^{+0.16}$ & $1.9 \times 10^{-12}$ \\
$41152-70835$ & & $1.72_{-0.21}^{+0.21}$ & $2.1 \times 10^{-12}$ \\
$73793-89232$ & & $1.78_{-0.68}^{+0.70}$ & $6.6 \times 10^{-13}$ \\
\hline
\end{tabular}

\# The $3 \sigma(\Delta \chi=6.43)$ upper limit is $2.0 \times 10^{21} \mathrm{~cm}^{-2}$. Value fixed for all the four PC observations. The $3 \sigma$ upper limit is $1.2 \times 10^{21} \mathrm{~cm}^{-2}$. * Adding a cut-off to the power law, the photon index is constrained to be $3.03_{-2.11}^{+0.80}$ and the high energy cut-off $>0.5 \mathrm{keV}$.

\section{Discussion}

GRB 050724 has a rich temporal and spectral phenomenology. The first peak in the BAT data is hard and typical of the short/hard GRB population. Long-soft emission following the hard peak has been reported by Lazzati et al. (2001), similarly to what is observed here for GRB 050724. Moreover, GRB 050709 discovered by HETE-2 (Villasenor et al. 2005) shows a similar soft bump in the Soft X-ray Camera (SXC) and a late flare (Fox et al. 2005). We can speculate that these bumps are a common characteristic of at least some of the short GRBs. This is at variance with at least three other short/hard GRBs: GRB 050509B (Gehrels et al. 2005), GRB 050813 (Retter et al. 2005) and GRB 050906 (Krimm et al. 2005), that do not show signs of extended emission (GRB 050509B has only 11 photons collected by XRT within $1000 \mathrm{~s}$ ). These characteristics may suggest two classes of short GRBs: one with strong soft emission following the hard short peak and the other with very faint afterglows. We can speculate that this second class might be related to Soft Gamma-ray Repeaters (SGR) in closeby galaxies (and in fact the estimated redshift of the galaxies positionally closer to the these GRBs are lower than the ones showing soft bumps) even if the total energy must be an order of magnitude larger than the brightest flare observed to date from SGR 1806-20 (Dec 27, 2004; e.g. Palmer et al. 2005). Alternatively, short GRBs may exist with no bumps, as already observed for long GRBs and SGRs comprising a very small population $(\$ 1 \%)$. Weak afterglows might also be produced by a compact object (black hole - neutron star) mergers with later outbursts due to a longer disk lifetime (Barthelmy et al. 2005; Page et al. 2006; Davies et al. 2005) or to bursts occurring in low density environment (Vietri 1997; Page et al. 2006).

The most striking feature in the afterglow of GRB 050724 are the big flares and the extended emission following the short initial spike. The first two flares have been modelled both as two Gaussians and two FRED-like bursts (with the largest portion of the energy released in the first one). The total $(0.2-10 \mathrm{keV})$ energy of the first flare is $6 \times 10^{49} \mathrm{erg} \mathrm{s}^{-1}$. If alone it might be consistent with the model by MacFadyen et al. (2005) suggesting that these flares arise from the interaction of the GRB outflow with a non-compact stellar companion. However, the presence of multiple bumps (and in particular the second one) strongly argue against this interpretation. In addition, these bumps have short durations $(\delta t / t<1)$, implying that they originate in a region more compact than the external shock. Delayed activity from 
the inner engine is a more plausible explanation (Burrows et al. 2005b; Romano et al. 2006; Falcone et al. 2006).

A different approach (even if less detailed) might rely on the self-gravitating fragments of (unstable) neutronized matter left over during a binary neutron star coalescence due to sausage instability (Faber et al. 2006; Colpi \& Rasio 1994; see also Perna et al. 2006). An interesting feature of the first flare is that its tail is characterized by peculiarly soft emission, softer than the late time emission dominated by the external shock component. Analogously to what is observed in long GRBs (Burrows et al. 2005b; Zhang et al. 2006; Lazzati \& Begelman 2006) the best explanation for the steep decay of the early afterglow is large angle emission from the internal shock phase. Such an emission component is expected to be very soft due to the large redshift implied by the relativistic de-beaming. This is an additional piece of evidence that the first bump is related to the inner engine and not to the interaction with a distant companion star.

The last two flares are less energetic $\left(6 \times 10^{47}\right.$ erg and $2 \times$ $\left.10^{49} \mathrm{erg}\right)$. These two flares may be the result of the interaction of the first ejecta (the one relative to the prompt emission of the short GRB), with the delayed ejecta of the two secondary flares. In particular, we note that the intensity ratio of the two early flares is similar to that of the two late flares.

Finally, the absence of any break in the light curve $\left(t_{\mathrm{b}}>\right.$ $3.5 \times 10^{5} \mathrm{~s}$ ) indicates that we have a large opening angle (although we caution the reader that the possible presence of flares makes the interpretation of the light curve in terms of power laws less secure). Under the simplifying assumption of a constant circumburst density medium of number density $n$, a fireball emitting a fraction of its kinetic energy in the prompt $\gamma$-ray phase would show a break in its afterglow light curve when its bulk Lorentz factor $\Gamma_{\mathrm{b}}$ becomes of the order of $\Gamma_{\mathrm{b}}=1 / \theta$, with $\theta=0.161\left(t_{\mathrm{b}, 1 \mathrm{~d}} /(1+z)\right)^{3 / 8}\left(n E_{\mathrm{iso}, 52}^{-1}\right)^{1 / 8}$ (with $E_{\mathrm{iso}, 52}$ the isotropic energy in units of $10^{52} \mathrm{erg}$ and $t_{\mathrm{b}, 1 \mathrm{~d}}$ the break time in units of 1 d; Rhoads 1997; Sari et al. 1999). In the case of GRB 050724 the isotropic total energy is $E_{\text {iso }} \sim 10^{51} \mathrm{erg}$ (a lower value would increase the angle even more) and for a standard density of 1 particle per $\mathrm{cm}^{-3}$, we obtain $\theta \gtrsim 20^{\circ}$, a very large value compared to long GRBs. In order to have a smaller opening angle $\left(\theta \lesssim 10^{\circ}\right)$ we would need either a lower particle density $n \lesssim 3 \times 10^{-3} \mathrm{~cm}^{-3}$ (or a break time hidden by the last flare, i.e. $t_{\mathrm{b}} \lesssim 70000 \mathrm{~s}$ ).

\section{Conclusion}

GRB 050724 is the first short GRB showing strong flaring and extended emission activity following short initial spike. This flaring activity has been collectively revealed by averaging short GRB light curves detected by BATSE in the 50-100 s time interval (Lazzati et al. 2001). This indicates a posteriori that this extended activity is likely a common characteristic of a good fraction of short GRBs. Moreover, these observations prove that flaring activity is a characteristic not just related to long GRBs (e.g. Burrows et al. 2005b) but to the GRB phenomenon itself.

We observe flaring activity both in the first stages of the Xray afterglow (i.e. within a few hundred seconds from the GRB onset) and at late times. Energetics (30\% of the afterglow fluence) and duration $(\delta t / t<0.4)$ considerations point toward a prolonged engine activity as already invoked for long GRBs (Burrows et al. 2005b; Falcone et al. 2006; Romano et al. 2006).
Finally, the lack of any break in the X-ray light curve likely indicates either a large beaming angle or a low density medium surrounding the GRB and, therefore, a different progenitor from long GRBs.

Acknowledgements. This work is supported at OAB by funding from ASI on grant number I/R/039/04, at Penn State by NASA contract NAS5-00136 and at the University of Leicester by the PPARC on grant numbers PPA/G/S/00524 and PPA/Z/S/2003/00507. DL acknowledges support from NSF grant AST-0307502 and NASA Astrophysical Theory Grant NAG5-12035. We gratefully acknowledge the contributions of dozens of members of the Swift team, who helped make this Observatory possible.

\section{References}

Barthelmy, S. D., Barbier, L. R., Cummings, J. R., et al. 2005a, Space Sci. Rev., 120,143

Barthelmy, S. D., Chincarini, G., Burrows, D. N., et al. 2005b, Nature, 438, 994 Bloom, J. S., Prochaska, J. X., Pooley, D., et al. 2006, ApJ, 638, 354

Burrows, D. N., Hill, J. E., Nousek, J. A., et al. 2005a, Space Sci. Rev., 120, 165 Burrows, D. N., Romano, P., Falcone, A., et al. 2005b, Science, 309, 1833 Chester, M., Covino, S., Schady, P., Roming, P., \& Gehrels, N. 2005, GCN 3670 Chincarini, G., Moretti, A., Romano, P., et al. 2005, ApJ, submitted [arXiv: astro-ph/0506453]

Colpi, M., \& Rasio, F. A. 1994, MmSAI, 65, 379

Covino, S., Antonelli, L. A., Romano, P., et al. 2005, GCN, 3665

Covino, S., Malesani, D., Israel, G. L., et al. 2006, A\&A, 447, L5

D’Avanzo, P., Covino, S., Antonelli, L. A., et al. 2005, GCN, 3690

Davies, M. B., Levan, A. J., \& King, A. R. 2005, MNRAS, 356, 54

Faber, J. A., Baumgarte, T. W., Shapiro, S. L., Taniguchi, K., \& Rasio, F. A. 2006, Phys. Rev. D, 73, 024012

Falcone, A. D., Burrows, D. N., Lazzati, D., et al. 2006, ApJ, 641, 1010

Fox, D. A, Frail, D. A., Price, P. A., et al. 2005, Nature, 437, 845

Gal-Yam, A., Cenko, S. B., Berger, E., Krzeminski, W., \& Lee, B. 2005, GCN, 3681

Gehrels, N., Chincarini, G., Giommi, P., et al. 2004, ApJ, 611, 1005

Gehrels, N., Sarazin, C. L., O'Brien, P. T., et al. 2005, Nature, 437, 851

Gladders, M., Berger, E., Morrell, N., \& Roth, M. 2005, GCN, 3798

Hill, J. E., Burrows, D. N, Nousek, J. A., et al. 2004, SPIE, 5165, 217

Hjorth, J., Watson, D., Fynbo, J. P. U., et al. 2005, Nature, 437, 859

Kouveliotou, C., Meegan, C. A., Fishman, G. J., et al. 1993, ApJ, 413, L101

Krimm, H., Barbier, L., Barthelmy, S., et al. 2005, GCN, 3667

Lazzati, D., \& Begelman, M. C. 2006, ApJ, 641, 972

Lazzati, D., Ramirez-Ruiz, E., \& Ghisellini, G. 2001, A\&A, 379, L39

MacFadyen, A. I., Ramirez-Ruiz, E., \& Zhang, W. 2005 [arXiv:astro-ph/0510192]

Moretti, A., Perri, M., Capalbi, M., et al. 2006, A\&A, 448, L9

Norris, J. P., \& Bonnell, J. T. 2006, ApJ, 643, 266

Nousek, J. A., Kouveliotou, C., Grupe, D., et al. 2006, ApJ, 642, 389

O'Brien, P. T. O., Willingale, R., Osborne, J., et al. 2006, ApJ, in press, [arXiv:astro-ph/0601125]

Page, K. L., King, A. R., Levan, A. J., et al. 2006, ApJ, 637, L13

Palmer, D. M., Barthelmy, S., Gehrels, N., et al. 2005, Nature, 434, 1107

Perna, R., Armitage, P. J., \& Zhang, B. 2006, ApJ, 636, L29

Prochaska, J. X., Bloom, J. S., Chen, H.-W., et al. 2005, GCN, 3700

Prochaska, J. X., Bloom, J. S., Chen, H.-W., et al. 2006, ApJ, 642, 989

Retter, A., Barbier, L., Barthelmy, S., et al. 2005, GCN, 3788

Romano, P., Moretti, A., Covino, S., et al. 2005a, GCN, 3669

Romano, P., Moretti, A., Vaughan, S., et al. 2005b, GCN, 3685

Romano, P., Moretti, A., Banat, P., et al. 2006, A\&A, in press [arXiv: astro-ph/0602497]

Roming, P. W. A., Kennedy, T. E., Mason, K. O., et al. 2005, Space Sci. Rev., 120,95

Soderberg, A. M. 2005, GCN, 3696

Soderberg, A. M., Cameron, P. B., \& Faril, D. A. 2005, GCN, 3684

Vaughan, S., Willingale, R., Romano, P., et al. 2006, ApJ, 639, 323

Vietri, M. 1997, ApJ, 478, L9

Villasenor, J. S., Lamb, D. Q., Ricker, G. R., et al. 2005, Nature, 437, 855

Zhang, B., Fan, Y. Z., Dyks, J., et al. 2006, ApJ, 642, 354 